\title{
Comparison of the Quantum-Chemical Models for the Calculation of Spectroscopic Properties of GeO
}

\author{
Vladimir V. Turovtsev, Yurij D. Orlov, \\ Mamikon Y. Gavalyan and Ivan A. Kaplunov* \\ Tver State University \\ 33 Zhelyabova, Tver, 170100, Russia
}

Received 19.04.2015, received in revised form 09.07.2015, accepted 12.15.2015

Calculations are made of the gaseous GeOvalent oscillation frequency in different quantum-mechanical models. Collation with experiment is given. A correlation is found between the calculated values of $\mathrm{r}(\mathrm{Ge}-\mathrm{O})$ relations with harmonic oscillation frequency. The parameters of the linear regression "harmonic frequency - bond length" are obtained. Calibration of the quantum-chemical methods for the calculation of $\mathrm{GeO}$ properties is carried out.

Keywords: germanium, quantum calculations, oxygen in germanium, "structure-property" correlation.

DOI: $10.17516 / 1999-494 X-2015-8-8-1056-1062$.

\section{Сравнение квантово-химических моделей расчета спектроскопических свойств $\mathrm{GeO}$}

\author{
В.В. Туровцев, Ю.Д. Орлов, \\ М.Ю. Гавалян, И.А. Каплунов \\ Тверской государственный университет \\ Россия, 170100, Тверь, Желябова, 33
}

В статье проведен расчет частоты валентного колебания газообразного $\mathrm{GeO}$ в различных квантово-химических моделях. Проведено сопоставление с экспериментом. Найдена корреляция между расчетными значениями длины связи $r(\mathrm{Ge}-\mathrm{O})$ и гармонической частотой колебания. Получены параметры линейной регрессии «гармоническая частота-длина связи». Проведена калибровка квантово-химических методов по расчету свойств GeO.

Ключевые слова: германий, квантовый расчет, кислород в германии, корреляции «строениесвойство».

(C) Siberian Federal University. All rights reserved

* Corresponding author E-mail address: Ivan.Kaplunov@tversu.ru 


\section{Введение}

Развитие элементной базы промышленности, связанной с электроникой, микроэлектроникой, фотоникой, требует получения и использования чистых, особо чистых веществ, а также совершенных кристаллических материалов. Кристаллический германий используется в полупроводниковой электронике при изготовлении элементов микросхем, отдельных радиоэлектронных компонентов, в качестве фотоприемников, как детекторный материал для ионизирующих излучений $[1,2]$. Германий широко применяют в инфракрасной оптике в качестве материала линз и окон, в акустооптике - при изготовлении модуляторов инфракрасного диапазона $[3,4]$. Как подложечный и одновременно функциональный материал германий используют для производства солнечных элементов с высоким КПД [2].

В ряде случаев возможно использование кристаллов без особых требований к структуре (оптический германий), но высокую чистоту и совершенную структуру должен иметь детекторный германий, а подложки для фотоэлектрических преобразователей, в свою очередь, должны иметь низкую плотность структурных дефектов. Возникновение дефектов кристаллической структуры происходит по различным причинам, основная из которых тепловые условия выращивания (как следствие - термоупругие напряжения). Одновременно существенное влияние на получение бездислокационного германия и особочистого германия оказывает присутствие кислорода в растущем кристалле. Кислород в германии определяет динамику образования дислокаций, микродефектов, термодоноров, влияет на время жизни неравновесных носителей заряда; на кислородных облаках, активированных легирующей примесью, происходит рассеяние излучения [5-7].

Анализ состояния кислорода в кристаллическом германии и оценка его влияния на свойства материала представляет собой актуальную задачу. Решение данной задачи следует искать в виде построения сначала классической (феноменологической), а потом и квантовой моделей сорбции и десорбции кислорода кристаллами.

Корректное описание физических явлений должно опираться на некоторую основу в виде физико-математической модели. Постановка и проведение эксперимента по изучению поведения кислорода в германии - довольно сложная и затратная процедура [5-7]. Поэтому желательно, с одной стороны, предварять все предполагаемые измерения теоретическим прогнозом, а с другой - подтверждать возникающие в результате исследований гипотезы теоретическими (в том числе численными) построениями. Целью настоящей работы была как оценка частоты валентного колебания в газообразном $\mathrm{GeO}$ в различных квантово-химических моделях (набор метод/базис), так и калибровка этих квантово-химических моделей.

\section{Анализ частоты валентного колебания \\ и длины связи оксида германия}

Моделирование термодинамических свойств веществ (температурный сдвиг энтальпии $H_{T}^{0}-H_{0}^{0}$, температурный сдвиг свободной энергии Гиббса $G_{T}^{0}-H_{0}^{0}$, энтропия $S^{0}$, теплоемкость $C_{p}^{0}$, энтальпия $\Delta_{f} H_{T}^{0}$ и свободная энергия Гиббса $\Delta_{f} G_{T}^{0}$ образования из простых веществ) в приближении разделения движений приводит к представлению этих свойств в виде суммы составляющих (вкладов). В таком формализме основную погрешность в итоговое значение свойства вносят электронное (полная электронная энергия) и колебательное движения. 
Вклад последнего движения зависит от геометрического строения соединения, массы атомов, частот колебаний и температуры. Ранее нами было проведено сравнение ошибок различных квантово-химических наборов метод/базис при воспроизведении длины связи $(r(\mathrm{Ge}-\mathrm{O}))$ в газообразном оксиде германия [8]. Молекула $\mathrm{GeO}$ была использована при калибровке моделей как наиболее простое кислородсодержащее соединение германия. Мы продолжили сопоставлять наиболее известные методы на примере расчета частоты валентного колебания связи $(\omega(\mathrm{Ge}-\mathrm{O}))$.

Отобранные из литературных источников частоты валентного колебания связи $\omega(\mathrm{Ge}-\mathrm{O})$ сведены в табл. 1 и 2. Спектр газообразного оксида германия содержит большое количество линий, которые при повышении температуры сливаются в широкие полосы. Сложность спектра связана с присутствием в природных образцах $\mathrm{GeO}$ нескольких изотопов обоих веществ, и в большинстве расчетных спектров была использована комбинация ${ }^{74} \mathrm{Ge}^{16} \mathrm{O}$, соответствующая природному изотопическому составу элементов.

Все рассмотренные нами теоретические частоты (табл. 2 и 3) были найдены в гармоническом приближении $\left(\omega_{\text {harm }}\right)$. Гармоническая частота также может быть получена и при рассмотрении экспериментальных спектров в рамках колебательной теории возмущений. В работе [9] $\omega_{\text {harm }}$ была положена равной $985.5 \mathrm{~cm}^{-1}$. В связи с различием в моделях (гармонический осциллятор - расчет и ангармонический осциллятор - эксперимент) теоретическая $\omega_{\text {harm }}$ не обязана совпадать с экспериментальной $\left(\omega_{\text {exp }}\right)$, в то же время близость $\omega_{\text {harm }}$ и $\omega_{\text {ехр }}$ свидетельствует об адекватности выбранного метода. Как следует из табл. 2, наибольшее согласие с экспериментом показали методы CCSD(T), CISD, MP2 и B3LYP. Однако во многих случаях даже для этой простой молекулы расхождение составило до 60 и более $\mathrm{cm}^{-1}$.

Ранее [10] было показано, что с увеличением числа базисных функций $\left(n_{b a s}\right)$ длина валентной связи, как правило, уменьшается, а с улучшением учета межэлектронной корреляции увеличивается. Это $\left(r=f\left(n_{b a s}\right)\right)$ неизбежно приводит к зависимости между размером базиса

Таблица 1. Экспериментальные частоты (волновые числа) $\mathrm{GeO}\left(\omega_{\text {exp }}\right)$ в разных агрегатных состояниях, $\mathrm{cm}^{-1}$

\begin{tabular}{|l|l|l|l|l|l|l|l|l|l|l|l|l|l|}
\hline Соединение & {$[9]$} & {$[9]^{1}$} & {$[14]$} & {$[15]$} & {$[16]$} & {$[17]$} & {$[18]$} & {$[19]^{2}$} & {$[19]^{3}$} & {$[20]^{1}$} & {$[21]$} & {$[22]^{4}$} & {$[23]^{4}$} \\
\hline${ }^{76} \mathrm{Ge}^{18} \mathrm{O}$ & & & 924 & & & & & & 926 & & & & 927 \\
\hline${ }^{74} \mathrm{Ge}^{18} \mathrm{O}$ & & & 926 & & & & & 929 & 928 & 925 & & & 930 \\
\hline${ }^{73} \mathrm{Ge}^{18} \mathrm{O}$ & & & 927 & & & & & & & & & & \\
\hline${ }^{72} \mathrm{Ge}^{18} \mathrm{O}$ & & & 928 & & & & & 931 & 930 & 928 & & & 932 \\
\hline${ }^{70} \mathrm{Ge}^{18} \mathrm{O}$ & & & 931 & & & & & 934 & 933 & 931 & & & 935 \\
\hline${ }^{76} \mathrm{Ge}^{16} \mathrm{O}$ & & & 969 & & & & & 973 & 971 & & & & 973 \\
\hline${ }^{74} \mathrm{Ge}^{16} \mathrm{O}$ & 978 & 986 & 971 & 975,1 & 985,5 & & & 975 & 973 & 970 & & & 975 \\
\hline${ }^{73} \mathrm{Ge}^{16} \mathrm{O}$ & & & 972 & & & & & 977 & 975 & & & & \\
\hline${ }^{72} \mathrm{Ge}^{16} \mathrm{O}$ & & 989 & 973 & & & & & 978 & 976 & 973 & & & 978 \\
\hline${ }^{70} \mathrm{Ge}^{16} \mathrm{O}$ & & 991 & 976 & & & & & 980 & 978 & 975 & & & 980 \\
\hline $\mathrm{Ge}^{16} \mathrm{O}^{5}$ & & & & 966 & & 976 & 976 & & & & 977 & 975.1 & 975.1 \\
\hline
\end{tabular}

${ }^{1} \omega_{\text {harm }},{ }^{2}$ в твердом $\mathrm{Ar},{ }^{3}$ в твердом $\mathrm{N}_{2},{ }^{4}$ твердый $\mathrm{GeO},{ }^{5}$ смесь изотопов $\mathrm{Ge}$. 
Таблица 2. Теоретические гармонические частоты (волновые числа) $\mathrm{GeO}\left(\omega_{\text {harm }}\right), \mathrm{cm}^{-1}$

\begin{tabular}{|l|l|l|l|}
\hline \multicolumn{1}{|c|}{ Метод/базис } & \multicolumn{1}{c|}{$\omega_{\text {harm }}$} & \multicolumn{1}{c|}{ Метод/базис } & $\omega_{\text {harm }}$ \\
\hline HF/VTZ & $1127[24]$ & CCSD(T)/SDB-cc-pVTZ & $925[29]$ \\
\hline HF/6-311G** & $1141[23]$ & CCSD(T)/cc-pVDZ-PP/RECP & $930[28]$ \\
\hline HF/DZP & $1143[23]$ & CCSD(T)/SDB+CPP-pVTZ & $931[29]$ \\
\hline HF/SVP & $1148[23]$ & CCSD(T)/SDB-cc-pVQZ & $943[29]$ \\
\hline HF/DZP+f & $1166[23]$ & CCSD(T)/SDB+CPP-pVQZ & $949[29]$ \\
\hline HF/SVP+f & $1176[23]$ & CCSD(T)/cc-pVTZ-PP/RECP & $980[28]$ \\
\hline Dirac-Hartree-Fock/VTZ & $1123[24]$ & CCSD(T)/cc-pV5Z-DK & $980[28]$ \\
\hline PT1/VTZ 1 & $1124[24]$ & CCSD(T)/cc-pVQZ-PP/RECP & $982[28]$ \\
\hline MP2/DZP & $944[23]$ & CCSD(T)/cc-pV5Z-PP/RECP & $982[28]$ \\
\hline MP2/TZVP & $944[14]$ & CISD/O - 10s6pld/[3s3pld] & $982[25]$ \\
\hline MP2/6-311G** & & Ge-5s5p2d & $993[27]$ \\
\hline MP2/SVP & $953[23]$ & B3LYP/6-311G* & $1001[23]$ \\
\hline MP2/DZP+f & $957[23]$ & B3LYP/DZP & $1006[23]$ \\
\hline MP2/SVP+f & $980[23]$ & B3LYP/6-311G** & $1010[23$ \\
\hline B3LYP/6-311++G(d,p) & $997[23]$ & B3LYP/SVP & $1010[14]$ \\
\hline B3LYP/6-311G & $975[17]$ & B3LYP/SVP & $1018[23]$ \\
\hline B3LYP/6-311+G(2df, 2pd) & $976[26]$ & B3LYP/DZP+f & $1030[23]$ \\
\hline
\end{tabular}

1 теория возмущений первого порядка.

и $\omega_{\text {harm }}$ при использовании одного и того же метода расчета ${ }^{1}$ (табл. 2). Поэтому наши расчеты, собранные в табл. 3, были выполнены с разными функционалами DFT, но с одним и тем же декартовым базисом 6-311++G(3df,3pd) 6d 10f.

Оптимизация равновесного строения и поиск $\omega_{\text {harm }}$ выполнены с помощью программ GAUSSIAN 09 [11]. Расчет гармонических частот $\omega_{\text {harm }}$ методами функционала плотности с базисом 6-311++G(3df,3pd) представлен в табл. 3. Выбор базиса обоснован в [10, 12]. Как следует из табл. 3, наиболее приемлемые результаты получены с функционалами mPW2PLYP, VSXC, XAlpha, SVWN и B3LYP. Расхождение составило не более $15 \mathrm{~cm}^{-1}$, что позволяет использовать найденные величины при расчетах термодинамических свойств газообразного $\mathrm{GeO}$ с удовлетворительной точностью [10].

Проведенное исследование (данная работа и [8]) дает возможность оценить применимость различных методов теории функционала плотности (в формализме Кона-Шема) к расчету геометрических и спектроскопических свойств $\mathrm{GeO}$ и выбрать среди них наиболее удовлетворяющий критерию согласия с экспериментом. Кроме того, это позволяет дать прогноз по расчету $\omega_{\text {harm }}$ на основании только геометрических параметров (модель Баджера [13]). Для этого нами была рассмотрена корреляции между $\omega_{\text {harm }}$ и $r(\mathrm{Ge}-\mathrm{O})$ и вычислены параметры линейной регрессии (1) «гармоническая частота-длина связи»:

$$
\omega_{\text {harm }}=\rho r+b .
$$

1 И с одними и теми же настройками в ключевых словах при выполнении квантово-химических расчетов. 
Параметры (1) для полученной совокупности значений найдены дважды (рис. 1):

a) в общем случае (т.е. по данным наших табл. 2 и 3 и табл. $1-3$ из [8]) $\rho=-2751.37 \mathrm{~cm}^{-1} \times \AA^{-1}$ и $b=5486 \mathrm{~cm}^{-1}\left(r_{\text {корр }}=-0.846\right)$,

б) только наши расчеты (наша табл. 3 и табл. 3 из [8]) $\rho=-2511.33 \mathrm{~cm}^{-1} \times \AA^{-1}$ и $b=5094 \mathrm{~cm}^{-1}$ $\left(r_{\text {корр }}=-0.966\right)$.

Корреляция между длиной связи и частотой валентного колебания известна для многих соединений [13]. Как правило, эта зависимость обычно не является линейной, однако полученный нами коэффициент корреляции Пирсона $\left(r_{\text {корp }}\right)$ говорит о линейности $\omega_{\text {harm }}=f(r)$. При этом указанная прямая проходит выше экспериментальной точки (на рисунке отмечена ромбом), т.е. используемые функционалы (в среднем) завышают либо $\omega_{\text {harm }}$, либо $r(\mathrm{Ge}-\mathrm{O})$. Так, подстановка в (1) экспериментальной длины $r(\mathrm{Ge}-\mathrm{O})=1.625 \AA \AA$ приводит к $\omega_{\text {harm }}=1015 \mathrm{~cm}^{-1}$ и $\omega_{\text {harm }}=1013 \mathrm{~cm}^{-1}$ в случаях а) и б) соответственно.

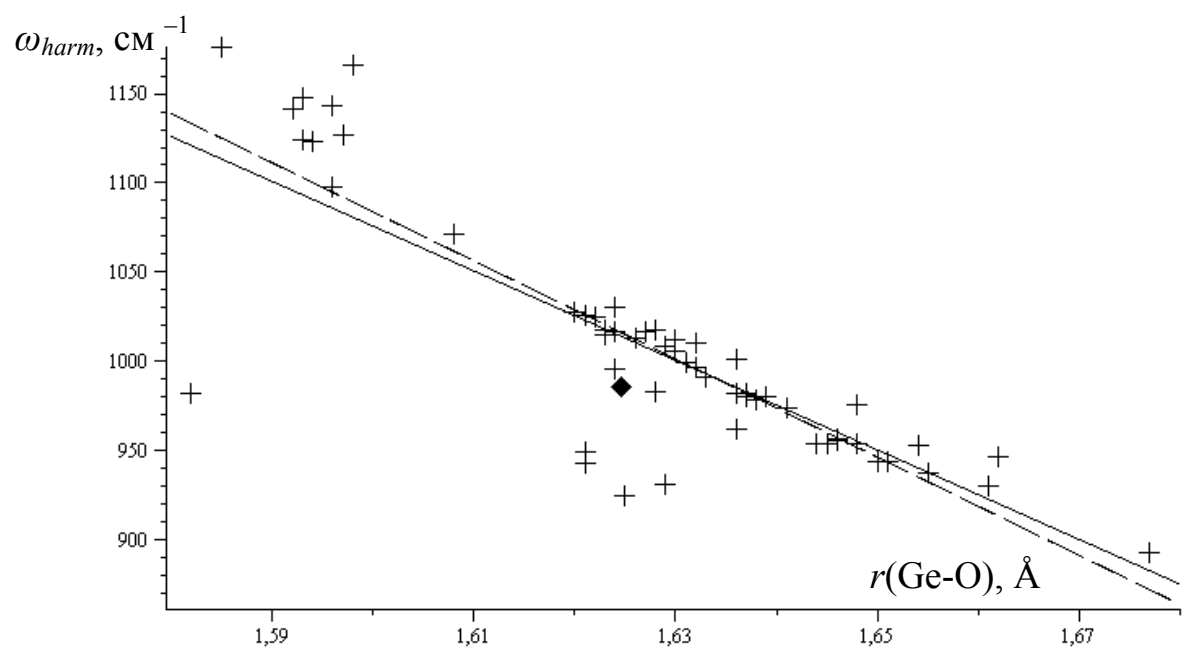

Рис. 1. Корреляционное поле и регрессии «гармоническая частота-длина связи». Сплошная линия построена по данным табл. 3, штриховая линия - по данным табл. 2 (см. в тексте). Ромбом отмечено экспериментальное значение

Таблица 3. Теоретические гармонические частоты (волновые числа) $\mathrm{GeO}\left(\omega_{\text {harm }}\right)$, см $^{-1}$

\begin{tabular}{|l|l|l|l|l|l|l|l|}
\hline \multicolumn{1}{|c|}{ Метод } & \multicolumn{1}{c|}{$\omega_{\text {harm }}$} & \multicolumn{1}{c|}{ Метод } & \multicolumn{1}{c|}{$\omega_{\text {harm }}$} & \multicolumn{1}{c|}{ Метод } & $\omega_{\text {harm }}$ & \multicolumn{1}{c|}{ Метод } & $\omega_{\text {harm }}$ \\
\hline HFB & 893 & VSXC & 978 & B972 & 1015 & BHandHLYP & 1071 \\
\hline BLYP & 937 & XAlpha & 983 & B98 & 1017 & BHandH & 1098 \\
\hline HFS & 954 & SVWN & 996 & B3P86 & 1017 & UMP2-FC & 947 \\
\hline B2PLYP & 954 & B3LYP & 999 & M06 & 1018 & MP2(FULL) & 954 \\
\hline BPW91 & 956 & B1LYP & 1009 & PBE1PBE & 1025 & & \\
\hline HCTH & 962 & B971 & 1012 & MPW1PW91 & 1026 & & \\
\hline mPW2PLYP & 974 & B3PW91 & 1013 & B1B95 & 1028 & $\omega_{\text {harm }}[11]$ & 985,5 \\
\hline
\end{tabular}


Если рассмотреть небольшую окрестность около экспериментальной точки (закрашенный черным ромб на рис. 1), то среди всех использованных моделей стоит выделить SVWN и XAlpha, которые удовлетворительно воспроизводят выбранные нами параметры и могут быть рекомендованы к расчету других свойств GeO. Большую погрешность к одновременному расчету реперных значений дают B972, B3PW91, B1LYP и B3P86. Однако сделанные предварительные выводы нуждаются в дополнительной проверке на геометрических и спектроскопических характеристиках других газообразных соединений германия (например, $\mathrm{GeO}_{2}$ ), а затем и при моделировании твердого тела.

\section{Заключение}

В работе проведена калибровка квантово-химических моделей по воспроизведению частоты валентного колебания и длины связи в газообразном оксиде германия. Наилучшее согласие с экспериментом и для частоты, и для длины связи показали функционалы SVWN, XAlpha, B972, B3PW91, B1LYP и B3P86, при этом если искать параметры по отдельности, то mPW2PLYP, VSXC, XAlpha, SVWN и B3LYP более точно воспроизводят частоту, а функционалы MB97-2, B972, B3LYP и LDA(SVWN), XAlpha. B3P86, B1B95, B1LYP, B98, B971, B3PW91, MPW1PW91, PBE1PBE, М06 - длину связи.

Авторы выражают благодарность Казанскому государственному технологическому университету за техническую поддержку при проведении расчетов.

Работа выполнена в рамках реализации ФЦП «Исследования и разработки по приоритетным направлениям развития научно-технологического комплекса России на 2014-2020 годы», соглашение 14.577.21.0004 (RFMEFI57714X0004).

\section{Список литературы}

[1] Sullivan R.M. Proceedings of SPIE - The International Society for Optical Engineering Window and Dome Technologies and Materials XI. "Window and Dome Technologies and Materials XI", Orlando, FL, 2009, 73020L.

[2] Claeys Cor L., Simoen E. Germanium-based technologies: from materials to devices. Berlin, Elsevier, 2007, $480 \mathrm{p}$.

[3] Смирнов Ю. М., Каплунов И. А. Материаловедение, 2004, 5, 48-52 [Smirnov Iu.M., Kaplunov I.A. Materials Science, 2004, 5, 48-52]

[4] Каплунов И. А., Колесников А. И., Шайович С. Л., Талызин И. В. Оптический журнал, 2005, 72(3), 51-56 [Kaplunov I.A., Kolesnikov A.I., Shaiovich S.L., Talyzin I.V. Journal of Optical Technology, 2005, 72(3), 51-56]

[5] Подкопаев О.И., Кулаковская Т.В., Шиманский А.Ф. и др. Журнал СФУ. Техника и технологии, 2013, 6(6), 674-679 [Podkopaev O.I., Kulakovskaia T.V., Shimanskii A.F. et. al. J. Sib. Fed. Univ. Eng. technol., 2013, 6(6), 674-679]

[6] Подкопаев, О.И., Кулаковская, Т.В., Шиманский, А.Ф., и др. Журнал СФУ. Техника и технологии, 2012, 5(6), 631-637 [Podkopaev O.I., Kulakovskaia T.V., Shimanskii A.F. et. al. J. Sib. Fed. Univ. Eng. technol., 2012, 5(6), 631-637]

[7] Подкопаев О.И., Шиманский А.Ф. Выращивание монокристаллов германия с низким содержанием дислокачий и примесей. Красноярск: Сиб. федер. ун-т, 2013. 104 с. [Podkopaev 
O.I., Shimanskii A.F. Growing single crystals of germanium with a low content of impurities and dislocations, Krasnoyarsk, Sib. Fed. Univ., 2013, 104 p.]

[8] Туровцев В. В., Орлов Ю. Д., Каплунов И. А. Вестник ТвГУ. Сер. Химическая, 2015, 2, 12-18 [Turovtsev V.V., Orlov Iu.D., Kaplunov I.A. Bulletin of the Tver State University, Chemistry, 2015]

[9] Thompson G. A., Maki A. G., Weber A. J. Mol. Spectr., 1986, 116, 136-142.

[10] Туровцев В.В. Дисс ... д-ра. физ.-мат. наук. Тверь: ТвГУ. 2014. 373 с. [Turovtsev V.V. Doctor's dissertation of physical and mathematical sciences, Tver State University, 2014, 373 p.]

[11] Frisch M.J. et all. Gaussian 09, Revision C.01, Gaussian, Inc., Wallingford CT, 2010.

[12] Орлов М.Ю., Туровцев В.В., Орлов Ю.Д. Вест. Башкир. ун-та, 2008, 13(3/I), 758-760 [Orlov M.Iu., Turovtsev V.V., Orlov Iu.D. Bulletin of Bashkir University, 2008, 13(3/I), 758-760]

[13] Тюлин В.И. Колебательные и вращательные спектры многоатомных молекул. М.: Изд-во МГУ, 1987, 204 с. [Tiulin V.I. Vibrational and rotational spectra of polyatomic molecules, Moscow State University, 1987, 204 p.]

[14] Hassanzadeh P., Andrews L. J. Phys. Chem., 1992, 96(15), 6181 - 6185.

[15] Friesen M., Junker M., Schnoeckel H. J. Chem. Phys., 2000, 112(4), 1782-1784.

[16] Huber K., Herzberg G. Molecular Spectra and Molecular Structure. 4. Princeton, Van Nostrand, 1979.

[17] Teng Y.-L., Jiang L., Han S., Xu Q. J. Phys. Chem., 2007, 111(28), 6225-6231.

[18] Nefedov O. M. Pure and Appl. Chem., 1991, 63, 231-242.

[19] Ogden J. S., Ricks M. J. J. Chem. Phys., 1970, 52, 352-357.

[20] Withnall R., Andrews L. J. Phys. Chem., 1990, 94. P. 2351-2357.

[21] Boganov S. E., Faustov V. I., Khabashesku V. N., Kerzina Z. A., Kagramanov, N. D., et al. Rus. Chem. Bul., 1993, 42(4), 663-671.

[22] Zhou M., Shao L., Miao L. J. Phys. Chem., 2002, 106(27), 6483-6486.

[23] Zumbusch A., Schnoeckel H. J. Chem. Phys., 1998, 108(19), 8092-8100.

[24] Dyall K. G. J. Chem. Phys., 1993, 98(3), 2191-2197.

[25] Igel-Mann G., Stoll H., Preuss H. Mol. Phys., 1988, 65, 1329-1336.

[26] Johnson J.R. Tobias, Panas I. Chem. Phys., 1999, 249(2-3), 273-303.

[27] Lin C.-L, Su M.-D., Chu S.-Y. Chem. Phys., 1999, 249(2-3), 145-160.

[28] Peterson K. A. J. Chem. Phys., 2003, 119(21), 11099-11112.

[29] Martin J. M. L., Sundermann A. J. Chem. Phys., 2001, 114(8), 3408-3420. 